\title{
Significance of serum tumor necrosis factor-related apoptosis-inducing ligand as a prognostic biomarker for renal cell carcinoma
}

\author{
DAISUKE TOIYAMA $^{1}$, NATSUKI TAKAHA ${ }^{1}$, MASAHIDE SHINNOH ${ }^{1}$, TAKASHI UEDA $^{1,2}$, \\ YASUNORI KIMURA ${ }^{1}$, TERUKAZU NAKAMURA ${ }^{1}$, FUMIYA HONGO $^{1}$, KAZUYA MIKAMI $^{1}$, \\ KAZUMI KAMOI ${ }^{1}$, AKIHIRO KAWAUCHI ${ }^{1}$ and TSUNEHARU MIKI ${ }^{1,2}$ \\ Departments of ${ }^{1}$ Urology and ${ }^{2}$ Translational Cancer Drug Development, Kyoto Prefectural \\ University of Medicine, Kawaramachi-Hirokoji, Kyoto 602-8566, Japan
}

Received June 24, 2012; Accepted September 28, 2012

DOI: $10.3892 / \mathrm{mco} .2012 .35$

\begin{abstract}
Tumor necrosis factor-related apoptosis-inducing ligand (TRAIL) is endogenously expressed in immune cells and contributes to immunosurveillance for cancer. TRAIL induces apoptosis preferentially in various cancer cells, including renal cell carcinoma (RCC) cells. In this study, the serum TRAIL level was examined using an enzyme-linked immunosorbent assay in 52 healthy controls and in 84 RCC patients prior to surgery and its significance as a biomarker was evaluated. The median serum TRAIL level was lower in RCC patients compared to the healthy controls (55.9 vs. $103.1 \mathrm{pg} / \mathrm{ml} ; \mathrm{P}=0.019)$. RCC with lymph node metastasis (N1-2), distant metastasis (M1), stage III-IV, or microscopic venous invasion was associated with decreased serum TRAIL levels $(\mathrm{P}=0.032,0.067,0.020$ and 0.011$)$. When comparing serum TRAIL levels in the same RCC patients prior and subsequent to surgery $(\mathrm{n}=11)$, the levels were significantly higher after surgery $(\mathrm{P}=0.031)$. The cause-specific survival rate was significantly higher in RCC patients with high serum TRAIL levels compared to those with low serum TRAIL levels ( $\mathrm{P}=0.0451)$. TRAIL was estimated to contribute 64 and $13 \%$ of the lymphocyte-mediated cytotoxicity against human RCC ACHN and Caki-1 cells, respectively. These data suggest
\end{abstract}

Correspondence to: Dr Natsuki Takaha, Department of Urology, Kyoto Prefectural University of Medicine, Kawaramachi-Hirokoji, Kyoto 602-8566, Japan

E-mail: ntakaha@koto.kpu-m.ac.jp

Abbreviations: TNF, tumor necrosis factor; TRAIL, tumor necrosis factor-related apoptosis-inducing ligand; RCC, renal cell carcinoma; DR, death receptor, DcR, decoy receptor; ELISA, enzyme-linked immunosorbent assay

Key words: tumor necrosis factor-related apoptosis-inducing ligand, serum, biomarker, renal cell carcinoma that the serum TRAIL level may be useful as a prognostic biomarker in RCC patients.

\section{Introduction}

A balance between pro-apoptotic and survival signals is essential for the development and progression of cancer cells. Elucidation of the molecular mechanisms underlying these two signaling systems in various types of cancer and identification of useful biomarkers is likely to contribute to the establishment of novel diagnostic and therapeutic modalities. At present, three major ligand/receptor systems, Fas ligand/Fas (CD95L/ CD95), tumor necrosis factor (TNF)- $\alpha / \mathrm{TNF}$ receptor-1, and TNF-related apoptosis-inducing ligand (TRAIL)/TRAIL receptors, have been identified as apoptotic mechanisms (1). Of these, TRAIL has gained attention because it induces apoptosis preferentially in cancer rather than normal cells (2). This phenomenon can be explained by the differential expression of agonistic receptors in cancer, and dominant-negative antagonistic receptors in normal cells. Death receptors 4 (DR4) and 5 (DR5) (also known as TRAIL-R1 and TRAIL-R2, respectively) are agonistic receptors for TRAIL and transmit a pro-apoptotic signal to the caspase signaling cascade, leading to cell death $(3,4)$. On the other hand, decoy receptors 1 (DcR1) and 2 (DcR2) (also known as TRAIL-R3 and TRAILR4, respectively) are antagonistic receptors for TRAIL. Even when bound by TRAIL, they do not induce apoptosis due to their lacking an intracellular death domain $(4,5)$.

Renal cell carcinoma (RCC) may be treated by surgery when diagnosed at an early stage, whereas advanced RCC diagnosed as a disseminated disease cannot be treated by surgery alone. Cytokine therapy with interferon- $\alpha$ and interleukin- 2 has been used to treat metastatic RCC, but the objective response rate was only $10-20 \%(6,7)$. Molecular-targeted therapies using multiple tyrosine kinase and mTOR inhibitors were previously introduced for the treatment of metastatic RCC. Although these molecular-targeted drugs confer better survival, with an objective response rate of $30-40 \%$, a complete response is rarely achieved, and various side-effects occur (8-12). Therefore, novel therapeutic and diagnostic modalities are required for 
RCC. At present, no tumor marker or biomarker is used for $\mathrm{RCC}$ in clinical practice.

TRAIL is endogenously expressed in immune cells and contributes to immunosurveillance for cancer (13). It selectively induces apoptosis in various cancer cells, including RCC cells, in vitro and in vivo (14-17). In addition, a tissue microarray analysis of a large number of RCC cases demonstrated that high TRAIL-R2 and TRAIL, and low TRAIL-R4 expression levels in RCC tumor tissues were associated with worse disease-specific survival (18). These findings suggest that TRAIL/TRAIL receptors may be useful biomarkers in the diagnosis and treatment of RCC.

In this study, we examined the serum TRAIL level in RCC patients and healthy individuals and evaluated its significance as a biomarker. We also investigated the contribution of TRAIL to lymphocyte-meditated cytotoxicity against RCC cells in vitro. To the best of our knowledge, this is the first study investigating serum TRAIL levels in RCC patients.

\section{Materials and methods}

Patients. Peripheral blood samples were obtained from 84 patients with primary RCC prior to surgery or anticancer therapy. The tumors were removed by radical or partial nephrectomy. The patients were histopathologically diagnosed with RCC and comprised 59 males and 25 females, ranging in age from 32 to 85 years (median age, 62 years). The histopathological diagnosis confirmed 70 clear cell carcinomas, six papillary RCCs, two chromophobe and four spindle cell carcinomas and two unknown diagnoses. Their histological classifications and staging data according to the TNM classification system (International Union Against Cancer, 6th edition, 2002) were (n): T1 (55), T2 (8), T3 (18) and T4 (3); N0 (77), N1-2 (7); M0 (71) and M1 (13); stage I (52), stage II (8), stage III (11) and stage IV (13); and G1 (1), G2 (62) and G3 (21). Documented sites of metastasis were (n): lung (9), pleura (1), bone (1), brain (1), adrenal (1) and liver (1). Blood samples were also collected from 52 healthy donors with no history of malignant disease.

Serum was separated by centrifugation of the blood samples and was stored at $-80^{\circ} \mathrm{C}$ for future enzyme-linked immunosorbent assays (ELISAs). This study protocol was approved by our institutional review board. Informed consent was obtained from the participants.

Cell culture. ACHN and Caki-1 human RCC cell lines were used as targets. The cells were maintained in RPMI-1640 medium supplemented with $100 \mathrm{U} / \mathrm{ml}$ penicillin, $100 \mu \mathrm{g} / \mathrm{ml}$ streptomycin, and $10 \%$ fetal bovine serum, hereafter referred to as complete medium, at $37^{\circ} \mathrm{C}$ in a humidified atmosphere containing $5 \% \mathrm{CO}_{2}$.

Reagents. Recombinant human TRAIL was purchased from Peprotech EC (London, UK or Rocky Hill, NJ, USA). Monoclonal anti-human TRAIL/TNFS10 antibody (clone 75411) and human recombinant interferon- $\alpha$ were purchased from R\&D Systems, Inc. (Minneapolis, MN, USA).

ELISA for TRAIL. A sandwich ELISA was performed, according to the manufacturer's instructions (Invitrogen Corp.,
Carlsbad, CA, USA) to determine TRAIL levels in the sera of RCC patients and healthy donors. The TRAIL concentration measurements were calibrated against titration curves generated using reference standards. Repeated measurements yielded consistent results.

Patients were divided into two groups on the basis of serum TRAIL levels. Patients with high levels had serum TRAIL concentrations that exceeded the median value, and patients with low levels had serum TRAIL concentrations that were less than the median value.

Cytotoxicity assay. A crystal violet assay was used to determine tumor cell lysis, as previously described (19). Briefly, $100 \mu \mathrm{l}$ of the target cell suspension $\left(3.0 \times 10^{3}\right.$ cells $)$ were added to each well of 96-well flat-bottomed microtiter plates (Nunc, Roskilde, Denmark) and incubated for $12 \mathrm{~h}$ at $37^{\circ} \mathrm{C}$ in a humidified atmosphere of $5 \% \mathrm{CO}_{2}$. Various concentrations of human recombinant TRAIL in $100 \mu \mathrm{l}$ of complete medium, or lymphocyte suspension $\left(1.5 \times 10^{5}\right.$ cells $)$ in $100 \mu \mathrm{l}$ of complete medium with or without $80 \mathrm{ng} / \mathrm{ml}$ of anti-human TRAIL antibody were added to the wells, and the plates were incubated for $48 \mathrm{~h}$ at $37^{\circ} \mathrm{C}$ in a humidified atmosphere of $5 \%$ $\mathrm{CO}_{2}$. The culture medium was removed, and cell viability was evaluated by crystal violet staining, according to the manufacturer's instructions (Kishida Chemical Co., Ltd., Japan). In brief, the cells were fixed with glutaraldehyde, rinsed with water, and incubated with crystal violet. The plates were then rinsed with water and air-dried. The absorbance at $590 \mathrm{~nm}$ of each well was measured using a microculture plate reader (VersaMax; Molecular Devices, Sunnyvale, CA, USA). The percentage cytotoxicity was calculated as: percentage cytotoxicity $=[1-($ absorbance of experimental well/absorbance of control well)] x 100. Lymphocytes were prepared from the peripheral blood of normal controls, using Lymphoprep ${ }^{\mathrm{TM}}$ (Axis-Shield PoC AS, Oslo, Norway), according to the manufacturer's instructions.

Statistical analysis. The determinations were made in triplicate. Statistical analyses were performed using the $\mathrm{JMP}^{\circledR}$, version 8.0 (SAS Institute Inc., Cary, NC). The Kaplan-Meier survival method was used to generate survival curves and to calculate survival rates. Cause-specific survival was measured from the date of surgery to the date of cause-specific death or last follow-up. Differences between high and low serum TRAIL groups were evaluated using log-rank test for survival data. Wilcoxon rank-sum test and t-test were used for all other statistical analyses. $\mathrm{P}<0.05$ was considered to indicate a statistically significant difference.

\section{Results}

Circulating TRAIL levels in serum from healthy individuals and patients with RCC. ELISA was used to measure TRAIL levels in sera obtained from healthy control donors $(n=52)$ and patients with RCC $(n=84)$. The median serum TRAIL level in patients with RCC $(55.9 \mathrm{pg} / \mathrm{ml})$ was $\sim 54 \%$ of the level in healthy individuals $(103.1 \mathrm{pg} / \mathrm{ml})(\mathrm{P}=0.019)$ (Fig. 1A).

Serum TRAIL levels in patients with RCC based on clinicopathological factors. Serum TRAIL levels in patients with 
A

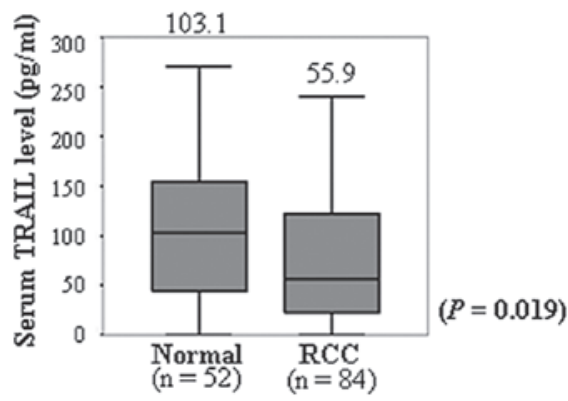

B

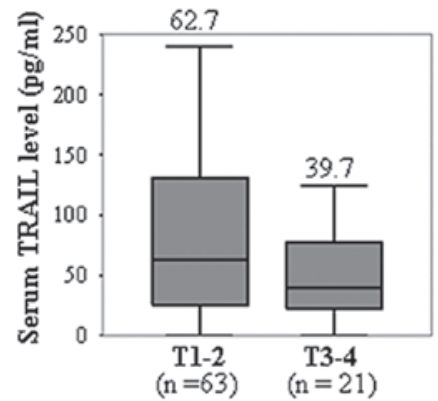

C

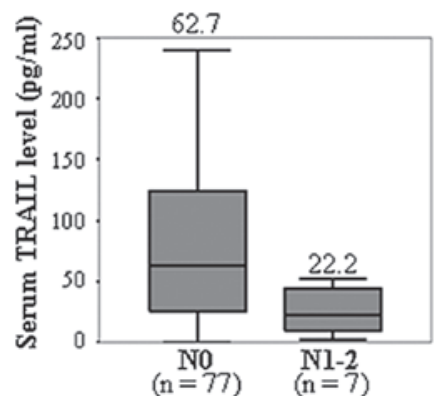

$(P=0.032)$

D

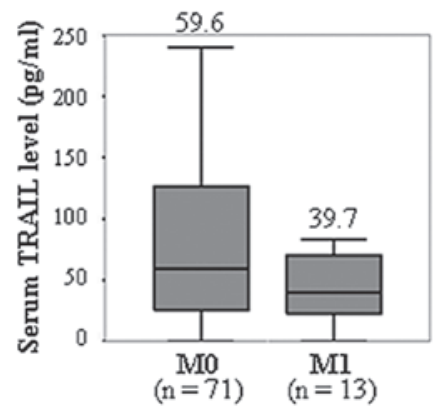

E

G
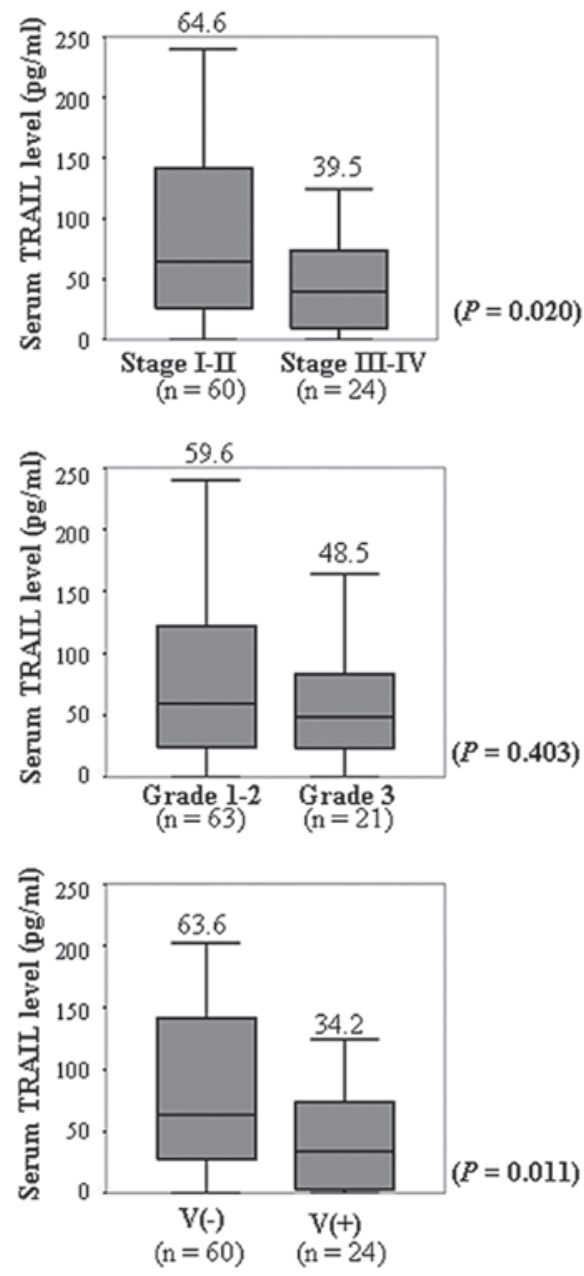

Figure 1. Correlation between serum TRAIL levels of RCC patients and clinicopathological factors is shown. The serum TRAIL levels were determined by ELISA. Boxplots are representative of the distribution of TRAIL levels in the sera of healthy controls or patients, based on clinicopathological factors. Statistical difference was analyzed using the Wilcoxon rank-sum test. The median serum TRAIL value of each group is shown above the box.

RCC were analyzed based on clinicopathological factors. Higher T classifications tended to be associated with lower serum TRAIL levels, thus patients classified as T3-4 had lower serum TRAIL concentrations compared to patients classified as T1-2. However, the difference was not statistically significant $(\mathrm{P}=0.083)$ (Fig. 1B). Serum TRAIL levels were markedly lower in RCC patients with lymph node metastasis (N1-2) compared to those without (NO; Fig. 1C; $\mathrm{P}=0.032$ ). Although serum TRAIL levels were lower in patients with distant metastasis (M1) than without (M0), the difference was not statistically significant (Fig. 1D) $(\mathrm{P}=0.067)$. RCC patients classified as stages III-IV had significantly lower serum TRAIL levels compared to patients classified as stages I-II (Fig. 1E) $(\mathrm{P}=0.020)$. The serum TRAIL level did not differ markedly in patients with Grade 3 RCC and those with Grades 1-2 (Fig. 1F; $\mathrm{P}=0.403)$. The serum TRAIL levels were significantly lower in RCC patients with microscopic venous invasion [ $\mathrm{V}(+)]$ compared to those without $[\mathrm{V}(-)]$ (Fig. $1 \mathrm{G} ; \mathrm{P}=0.011$ ). These findings suggest that the serum TRAIL level tends to be inversely correlated with the aggressiveness of RCC.

Correlation between the serum TRAIL level and postoperative cause-specific survival in RCC patients. The postoperative cause-specific survival of RCC patients who underwent complete resection of renal tumors by radical or partial nephrectomy was evaluated using the Kaplan-Meier method, while the correlation between the cause-specific survival and serum TRAIL level was analyzed using the 


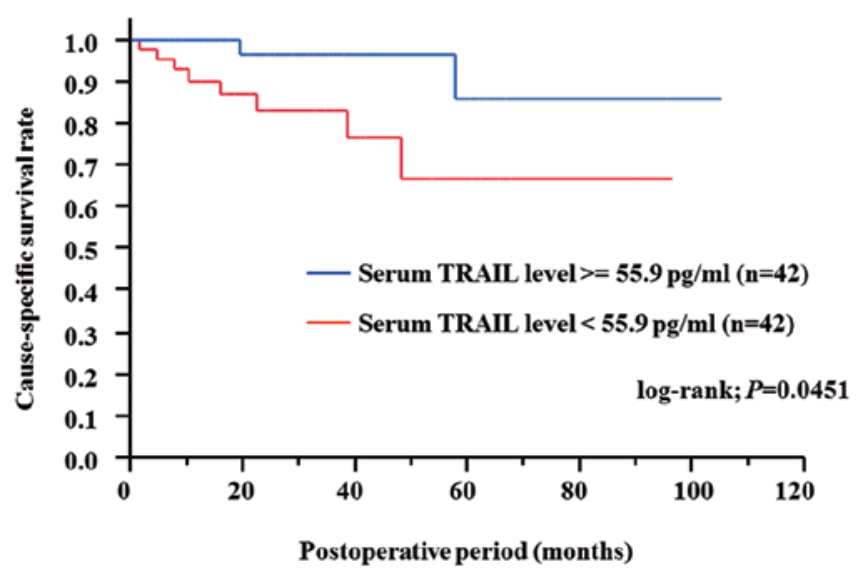

Figure 2. Kaplan-Meier curves of cause-specific survival based on the serum TRAIL level are shown. The patients were divided into two groups based on the median serum TRAIL value.

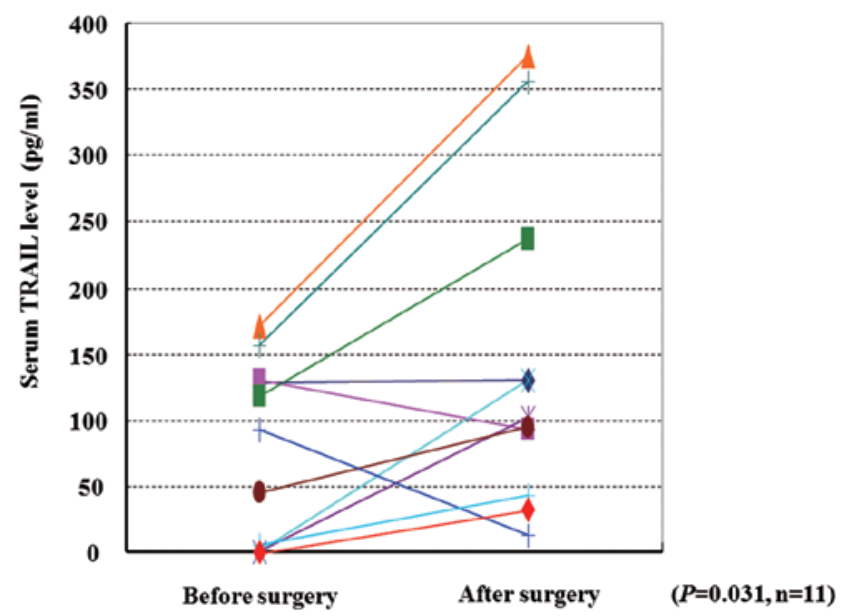

Figure 3. Comparison of the serum TRAIL levels in RCC patients before and after surgery is shown. Statistical difference was analyzed using the paired t-test.

log-rank test. For this analysis, RCC patients were divided into two groups: the high-serum TRAIL (i.e., TRAIL levels greater than the median value) and the low-serum TRAIL (i.e., TRAIL levels less than the median value) groups. The 3- and 5-year cause-specific survival rates were 83.1 and $66.6 \%$ in RCC patients with low serum TRAIL, and 96.3 and $85.6 \%$ in those with high serum TRAIL, respectively. The postoperative cause-specific survival rate was significantly higher in RCC patients with high serum TRAIL compared to patients with low serum TRAIL ( $\mathrm{P}=0.0451$, log-rank test) (Fig. 2). This finding suggests that the serum TRAIL level may be a useful prognostic marker for RCC patients.

Effect of surgical resection of RCC on circulating TRAIL levels in serum of RCC patients. For 11 RCC patients who did not have lymph node metastasis or distant metastasis (NOM0) at surgery, we compared the serum TRAIL levels in samples collected prior to and 2-4 weeks after surgery. The mean serum TRAIL levels prior and subsequent to surgery were $76.9 \pm 68.3$ and $145.8 \pm 124.4 \mathrm{pg} / \mathrm{ml}$, respectively. These levels were significantly different $(\mathrm{P}=0.031$, paired t-test) (Fig. 3). Thus, removal
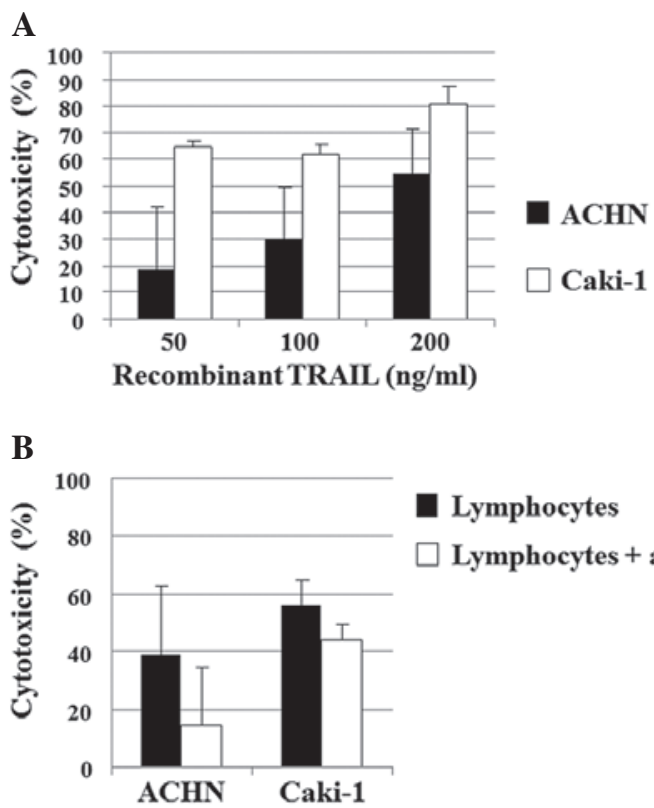

\section{Lymphocytes}

$\square$ Lymphocytes + anti-TRAIL Ab

Figure 4. (A) Cytotoxicity of human recombinant TRAIL against human RCC cell lines in vitro is shown. Cytotoxicity was examined by crystal violet assay after incubation for $48 \mathrm{~h}$. (B) Lymphocyte-mediated cytotoxicity against human RCC cell lines in vitro. Cytotoxicity was examined by crystal violet assay after incubation for $48 \mathrm{~h}$. To evaluate the contribution of TRAIL secreted by lymphocytes to the lymphocyte-mediated cytotoxic effect on RCC cells, cytotoxicity was examined in vitro in the presence of anti-human TRAIL antibody $(\mathrm{Ab})$.

of renal tumor may induce an increase in circulating serum TRAIL in RCC patients.

TRAIL-mediated cytotoxic effect of lymphocytes on RCC cells. Human recombinant TRAIL showed significant dose-dependent in vitro cytotoxicity against ACHN and Caki-1 cells, the two human RCC cell lines (Fig. 4A). Similarly, lymphocytes prepared from the healthy controls showed significant cytotoxicity against ACHN and Caki-1 cells in vitro (Fig. 4B). To evaluate the extent to which lymphocyte-secreted TRAIL contributed to the lymphocyte-mediated cytotoxic effect, cytotoxicity was examined in vitro in the presence of antihuman TRAIL antibody. The addition of anti-human TRAIL antibody reduced the cytotoxicity against ACHN and Caki-1 cells from 39 to $14 \%$ and 56 to $49 \%$, respectively (Fig. 4B). The contribution of lymphocyte-secreted TRAIL was estimated to be 64 and $13 \%$ of the total lymphocyte-induced cytotoxicity against ACHN and Caki-1 cells, respectively.

\section{Discussion}

Although cytokine and molecular-targeted therapies have been introduced for the treatment of metastatic RCC, their efficacy is unsatisfactory (6-12). Furthermore, no biomarkers for the diagnosis of RCC or the monitoring of treatment are available. Thus, useful biomarkers should be identified and novel diagnostic and therapeutic modalities for RCC patients, who are resistant to currently available therapeutic modalities, should be established.

The TRAIL/TRAIL receptor pathway is highly involved in inducing apoptotic signals $(1,14,16)$, as well as in maintaining 
immune surveillance potential (13) against cancer development and progression. The sensitivity of RCC cell lines to TRAIL has been studied with respect to the expression of TRAIL receptors and other signaling molecules, such as nuclear factor $\kappa$-light-chain-enhancer of activated B cells (NF- $\kappa \mathrm{B})(15) . \mathrm{In}$ addition, high TRAIL-R2 and TRAIL, and low TRAIL-R4 expression levels detected in surgical specimens by tissue microarray analysis have been reported to be associated with worse disease-specific survival in a large cohort of RCC patients (18). Thus, the utility of the serum TRAIL level as a biomarker for RCC was determined in this study. To the best of our knowledge, this is the first study investigating serum TRAIL levels in RCC patients as compared to levels in healthy individuals, and with regard to clinicopathological factors and postoperative prognosis.

We demonstrated markedly lower serum TRAIL levels in RCC patients compared to healthy control individuals and an inverse correlation between the serum TRAIL concentration and the progression and aggressiveness of RCC. These findings suggest that decreased TRAIL may allow cancer cells to escape immune surveillance and apoptosis, resulting in the development and progression of RCC. In addition, serum TRAIL levels were significantly elevated in RCC patients after complete surgical resection of the primary tumor. Based on this finding, we hypothesize that RCC tumors are involved in the inhibition of the production of TRAIL into the circulation via two possible mechanisms. First, RCC cells may secrete soluble factors that inhibit TRAIL production from immune cells, such as lymphocytes. The identification of these possible inhibitory soluble factors may provide a diagnostic tool and therapeutic target in RCC. The second possible mechanism involves sequestration of TRAIL-producing immune cells into renal tumors, resulting in decreased serum TRAIL levels. The high TRAIL expression detected in surgical RCC specimens by tissue microarray analysis (18) is consistent with this mechanism. With either of the possible mechanisms, removal of the renal tumor might result in the return of circulating TRAIL levels to baseline values. To this end, the circulating TRAIL level in sera of RCC patients may be a biomarker of postoperative progression or recurrence. Furthermore, we demonstrated that the cause-specific survival rate was significantly higher in RCC patients with high serum TRAIL levels, compared to RCC patients with low serum TRAIL levels. Taken together, our findings suggest that serum TRAIL might be useful as a prognostic biomarker of RCC. However, these hypotheses require confirmation by a larger-scale study comprising patients with a longer follow-up period.

A few previous studies have reported serum TRAIL levels in cancer patients. The serum TRAIL levels reported in patients with metastatic melanoma (20) and chronic myeloid leukemia (21) prior to interferon- $\alpha$ treatment were comparable to those in healthy donors. In their study, Perik et al (22) reported that post-chemotherapy serum TRAIL levels in long-term diseasefree breast cancer survivors were higher compared to those in healthy controls. Intensive immune surveillance for cancer cells by high serum TRAIL levels may be required for the long-term survival of advanced breast cancer patients. Thus, in certain types of cancer, including breast cancer and RCC, higher serum TRAIL levels may be associated with a better prognosis subsequent to definitive treatment, reflecting the potential of immune surveillance in limiting cancer development and progression. In the present study, TRAIL was estimated to contribute to $13-64 \%$ of the lymphocyte-mediated cytotoxic effect on RCC cells.

In conclusion, the present study suggests that the serum TRAIL level may be useful as a prognostic biomarker in RCC patients. One limitation of this study is the relatively small number of patients, thus these findings should be validated by studies conducted on a larger number of patients with longer follow-up times. Additional studies with a view to clarify the molecular mechanism underlying the inhibition of TRAIL release into the circulation in RCC patients are likely to facilitate the development of novel diagnostic and therapeutic modalities for RCC.

\section{Acknowledgements}

The authors would like to thank Ms. Yukako Morioka for the technical assistance and Dr Mano Horinaka for the critical reading and discussion of the manuscript.

\section{References}

1. Ashkenazi A and Dixit VM: Death receptors: Signaling and modulation. Science 281: 1305-1308, 1998.

2. Wiley SR, Schooley K, Smolak PJ, et al: Identification and characterization of a new member of the TNF family that induces apoptosis. Immunity 3: 673-682, 1995.

3. Pan G, O'Rourke K, Chinnaiyan AM, et al: The receptor for the cytotoxic ligand TRAIL. Science 276: 111-113, 1997.

4. Sheridan JP, Marsters SA, Pitti RM, et al: Control of TRAIL-induced apoptosis by a family of signaling and decoy receptors. Science 277: 818-821, 1997.

5. Pan G, Ni J, Wei YF, Yu G, Gentz R and Dixit VM: An antagonist decoy receptor and a death domain-containing receptor for TRAIL. Science 277: 815-818, 1997.

6. Mulders P, Figlin R, de Kernion JB, et al: Renal cell carcinoma: Recent progress and future directions. Cancer Res 57: 5189-5195, 1997.

7. Bex A, Haanen JB, Vyth-Dreese FA, Horenblas S and de Gast GC: Cytokine therapy response as a selection criterion for cytoreductive nephrectomy in metastatic renal clear-cell carcinoma of intermediate prognosis. Results and conclusions from a combined analysis. Urol Int 80: 367-371, 2008.

8. Escudier B, Eisen T, Stadler WM, et al: Sorafenib in advanced clear-cell renal-cell carcinoma. N Engl J Med 356: 125-134, 2007.

9. Hudes G, Carducci M, Tomczak P, et al: Temsirolimus, interferon alfa, or both for advanced renal-cell carcinoma. N Engl J Med 356: 2271-2281, 2007.

10. Motzer RJ, Escudier B, Oudard S, et al: Efficacy of everolimus in advanced renal cell carcinoma: a double-blind, randomised, placebo-controlled phase III trial. Lancet 372: 449-456, 2008.

11. Motzer RJ, Hutson TE, Tomczak P, et al: Sunitinib versus interferon alfa in metastatic renal-cell carcinoma. N Engl J Med 356: $115-124,2007$.

12. Froehner M, Hakenberg OW and Wirth MP: Molecular therapy in urologic oncology. Urol Int 79: 1-7, 2007.

13. Takeda K, Smyth MJ, Cretney E, et al: Critical role for tumor necrosis factor-related apoptosis-inducing ligand in immune surveillance against tumor development. J Exp Med 195: 161-169, 2002.

14. Ashkenazi A, Pai RC, Fong S, et al: Safety and antitumor activity of recombinant soluble Apo2 ligand. J Clin Invest 104: 155-162, 1999.

15. Oya M, Ohtsubo M, Takayanagi A, Tachibana M, Shimizu N and Murai M: Constitutive activation of nuclear factor-kappaB prevents TRAIL-induced apoptosis in renal cancer cells. Oncogene 20: 3888-3896, 2001.

16. Walczak H, Miller RE, Ariail K, et al: Tumoricidal activity of tumor necrosis factor-related apoptosis-inducing ligand in vivo. Nat Med 5: 157-163, 1999. 
17. Zeng Y, Wu XX, Fiscella M, et al: Monoclonal antibody to tumor necrosis factor-related apoptosis-inducing ligand receptor 2 (TRAIL-R2) induces apoptosis in primary renal cell carcinoma cells in vitro and inhibits tumor growth in vivo. Int J Oncol 28: 421-430, 2006.

18. Macher-Goeppinger S, Aulmann S, Tagscherer KE, et al: Prognostic value of tumor necrosis factor-related apoptosis-inducing ligand (TRAIL) and TRAIL receptors in renal cell cancer. Clin Cancer Res 15: 650-659, 2009.

19. Roux S, Apetoh L, Chalmin F, et al: $\mathrm{CD} 4{ }^{+} \mathrm{CD} 25^{+}$Tregs control the TRAIL-dependent cytotoxicity of tumor-infiltrating DCs in rodent models of colon cancer. J Clin Invest 118: 3751-3761, 2008.
20. Tecchio C, Huber V, Scapini P, et al: IFNalpha-stimulated neutrophils and monocytes release a soluble form of TNF-related apoptosis-inducing ligand (TRAIL/Apo-2 ligand) displaying apoptotic activity on leukemic cells. Blood 103: 3837-3844, 2004.

21. Tanaka H, Ito T, Kyo T and Kimura A: Treatment with IFNalpha in vivo up-regulates serum-soluble TNF-related apoptosis inducing ligand (sTRAIL) levels and TRAIL mRNA expressions in neutrophils in chronic myelogenous leukemia patients. Eur J Haematol 78: 389-398, 2007.

22. Perik PJ, Van der Graaf WT, De Vries EG, et al: Circulating apoptotic proteins are increased in long-term disease-free breast cancer survivors. Acta Oncol 45: 175-183, 2006. 